\title{
Relative motion at the bone-prosthesis interface
}

\author{
M Keja ${ }^{1}$, H W Wevers ${ }^{2}$, D Siu ${ }^{2}$, H Grootenboer ${ }^{1}$ \\ 'Department of Mechanical Engineering, University of Twente, Enschede, The \\ Netherlands; ${ }^{2}$ Clinical Mechanics Group, Department of Mechanical Engineering, and \\ Department of Surgery, Queen's University, Kingston, Ontario, Canada
}

\begin{abstract}
Summary
Bone ingrowth in porous surfaces of human joint implants is a desired condition for long-term fixation in patients who are physically active (such as in sport or work). It is generally recognized that little actual bone ingrowth occurs. The best clinical results report between 10 and $20 \%$ of the total prosthetic surface in contact with bone will feature good bone ingrowth. One inhibiting factor is the relative motion of the bone with respect to the implant during load-bearing. This study investigated mathematically the interface micromotion (transverse reversible relative motion) between a flat metal tibial prosthetic surface of a prototype implant, and the bone at the resection site. The aim was to assess the effect of perimeter fixation versus midcondylar pin fixation and the effect of plate thickness and plate stiffness.

Results showed that in the prototype design the largest reversible relative bone motion occurred at the tibial eminence. By design, the skirt fixation at the perimeter would prevent bone motion. A PCA (Howmedica Inc.) prosthesis has been widely used clinically and was chosen for a control because its fixation by two pegs beneath the condyles is a common variation on the general design of a relatively thick and stiff metal tibial support tray with pegs in each condylar area. The PCA tibial prosthesis showed the largest bone motion at the perimeter along the midcondylar mediolateral line, while being zero at the pegs. Maximum relative bone motion for the prototype was $37 \mu \mathrm{m}$ and for the control was $101 \mu \mathrm{m}$. Averaged values showed the prototype to have $38 \%$ of the relative reversible bone motion of the control (PCA).
\end{abstract}

\section{Relevance}

This work attempts to minimize (or control) the reversible bone motion that occurs at the implant-tissue interface by containing the lateral motion of the resected tibia while at the same time allowing the gross physiological bone motion of the proximal tibia. This is clinically relevant as the small bone-implant interface motion is more conducive to bone ingrowth and therefore improves fixation in biologically anchored implants.

Key words: Bone, interface, prosthesis, orthopaedic, motion

Clin. Biomech. 1994; 9: 275-283, September

\section{Introduction}

Total knee replacement (TKR)] has proved to be successful in older patients ${ }^{1}$. As prostheses were implanted in younger patients, long-term problems arose due to failure of the bonc-ccment interface ${ }^{2}$.

Received: 19 October 1992

Accepted: 4 November 1993

Correspondence and reprint requests to: Prof $\mathrm{H}$ W Wevers, Clinical Mechanics Group, Department of Mechanical Enginnering \& Department of Surgery, Queen's University, Kingston, Ontario, Canada K7L 2V7

(C) 1994 Butterworth-Heinemann Ltd

0268-0033-94-050275-09
These problems initiated a search for alternative fixation methods, such as hydroxyapatite and calcium phosphate coatings, porous coatings and press-fit systems ${ }^{3,4}$. Initial results with porous coatings were poor: bone ingrowth was limited, if present at all $^{5}$. Often fibrous tissue was formed between prosthesis and bone $^{5,6}$. Stress shielding was recognized as a possible inhibitor of bone ingrowth ${ }^{7.8}$ while excessively high stresses led to loosening of the tibial component ${ }^{9}$. In addition excessive relative motion between implant and bone was recognized as an important negative indicator even though bone ingrowth can occur in the presence of some very small movement (up to $28 \mu \mathrm{m}$ ) experimentally ${ }^{10,11}$. Lewis et al. ${ }^{9}$ compared six tibial 
component design configurations using finite element analyses and reported a single-post, metal-backed design provided the lowest system stresses overall. More specifically metal components provide lower bone stresses than polyethylene components and one-piece designs give lower bone-cement interface stresses compared with those with separated condylar components. The objective of this study was to compare the influence of different design parameters on the relative motion at the interface between tibia and tibial plateau implant. Two implants were used: the porous coated anatomical (PCA) tibial component (Howmedica Inc.) as a control and a new, more flexible tibial implant design (QUE).

Long-term irreversible relative motion, or migration, between prosthesis and bone is distinct from immediate reversible relative motion which occurs in reaction to load. The latter should be more important in the initial phase of bone remodelling, and therefore the purpose of this study is to quantify the reversible relative motion.

Several authors have tried to measure reversible relative motion. Ryd et al. measured it in vivo through roentgenstereophotogrammetric analysis $(\mathrm{RSA})^{6,12}$ while others studied it in vitro through direct measurement ${ }^{13}$. Although roentgenstereophotogrammetric analysis is an appropriate method it may not be accurate enough to measure motions smaller than $0.5 \mathrm{~mm}$ that may occur in reversible relative motion ${ }^{12}$. In Ryd's cases all reversible relative motion was thought to take place within radiolucent zones, representing the soft tissue of the interface ${ }^{6.12}$. Although this situation was not necessarily clinically unstable, it could not be considered ideal, and the magnitude of motion would be expected to be much larger than in the case of solid bone ingrowth.

In-vitro measurements of reversible relative motion in locations other than the free edges often involved damaging the bone and/or prosthesis. Most experimental methods have measured only a few points, making it difficult to show general trends. Also, difficulty in controlling surgical technique, loading conditions, and individual specimen variations made comparison of limited value. Furthermore, these methods were not suitable to analyse the effect of iterative changes in designs, as each experiment was time-consuming, unique, and expensive.

In orthopaedic research, finite element modelling (FEM) is a flexible tool for assessing parameters that are otherwise difficult to measure ${ }^{14}$. A choice must be made in FEM between two-dimensional (2D), axisymmetric, and three-dimensional (3D) modelling. Because of greater accuracy and simplicity at similar cost 2D or axisymmetric modelling is often preferred over $3 \mathrm{D}$ modelling ${ }^{14}$. The drawback of $2 \mathrm{D}$ and axisymmetric models is validity: most biomedical structures and loading conditions are three-dimensional and can only be roughly represented by a $2 \mathrm{D}$ model. $2 \mathrm{D}$ models $^{15}$ and axisymmetric models ${ }^{16}$ have been used to study reversible relative motion. However, since bone and prosthesis have intricate $3 D$ shapes a $3 D$ model was generated for our study. 3D models were reported by Strickland et al. ${ }^{17}$ and Natarajan and Andriacchi ${ }^{18}$. An attempt to validate a $3 D$ model for the calculation of relative motion was undertaken by Tissakht et al. ${ }^{13}$. They compared in-vitro measurements with a 3D FE model and found that the model predicted both trends and magnitudes of relative motions fairly well. Material properties of bone and FE models were not mentioned, especially the Poisson's ratio, which may have an effect on the stresses and strains.

Because bone is highly inhomogeneous and anisotropic, properties vary strongly from individual to individual and within each individual specimen ${ }^{19,20}$. This, and the limitations of FEM in general, made it desirable that the study be done only in a comparative and qualitative manner.

\section{Methods}

The ANSYS finite element program was used. The tibial geometry was a version of the model developed by Little et al. ${ }^{21}$ (Figure 1). In this work the proximal $100 \mathrm{~mm}$ of the tibia was modelled, since the influence

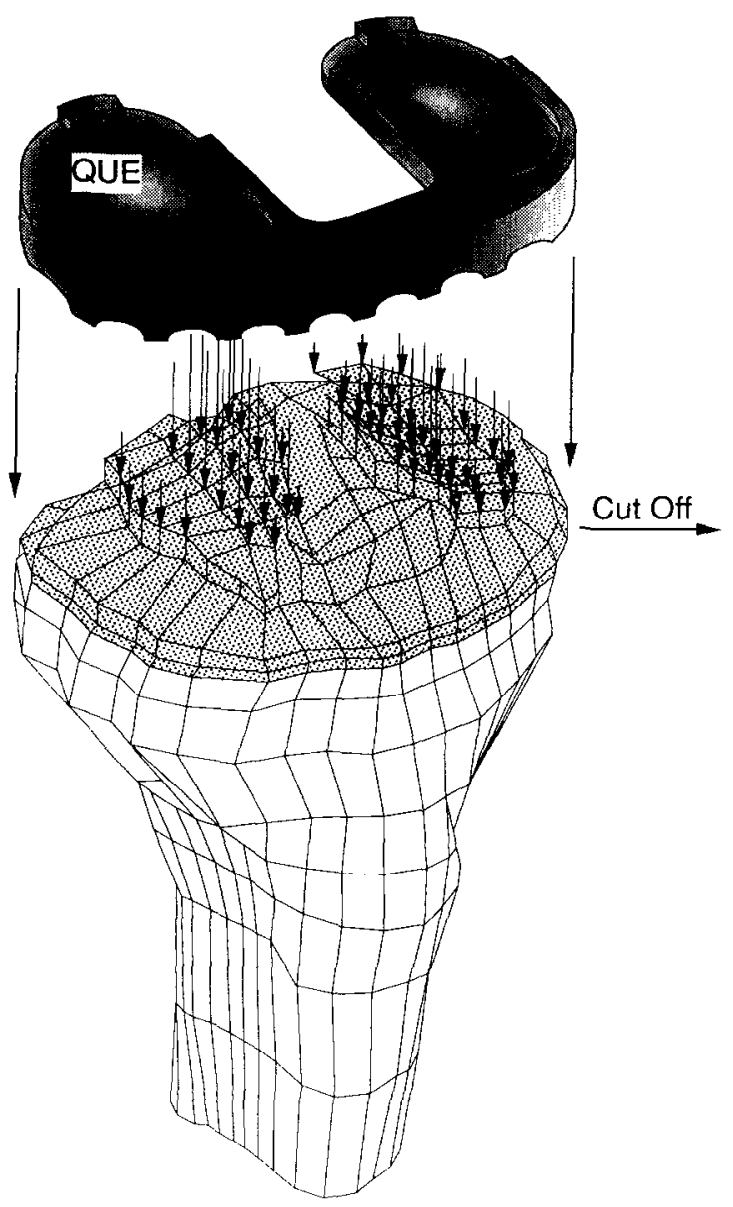

Figure 1. Tibial model by Little et al. ${ }^{21}$ used in this study. The top of the tibia ('cut off'), including the simulated cartilage load, has been removed to receive new implant and then only local modifications to the shape have been made to accommodate prosthetic fit 
TIBIA ASSEMBLY
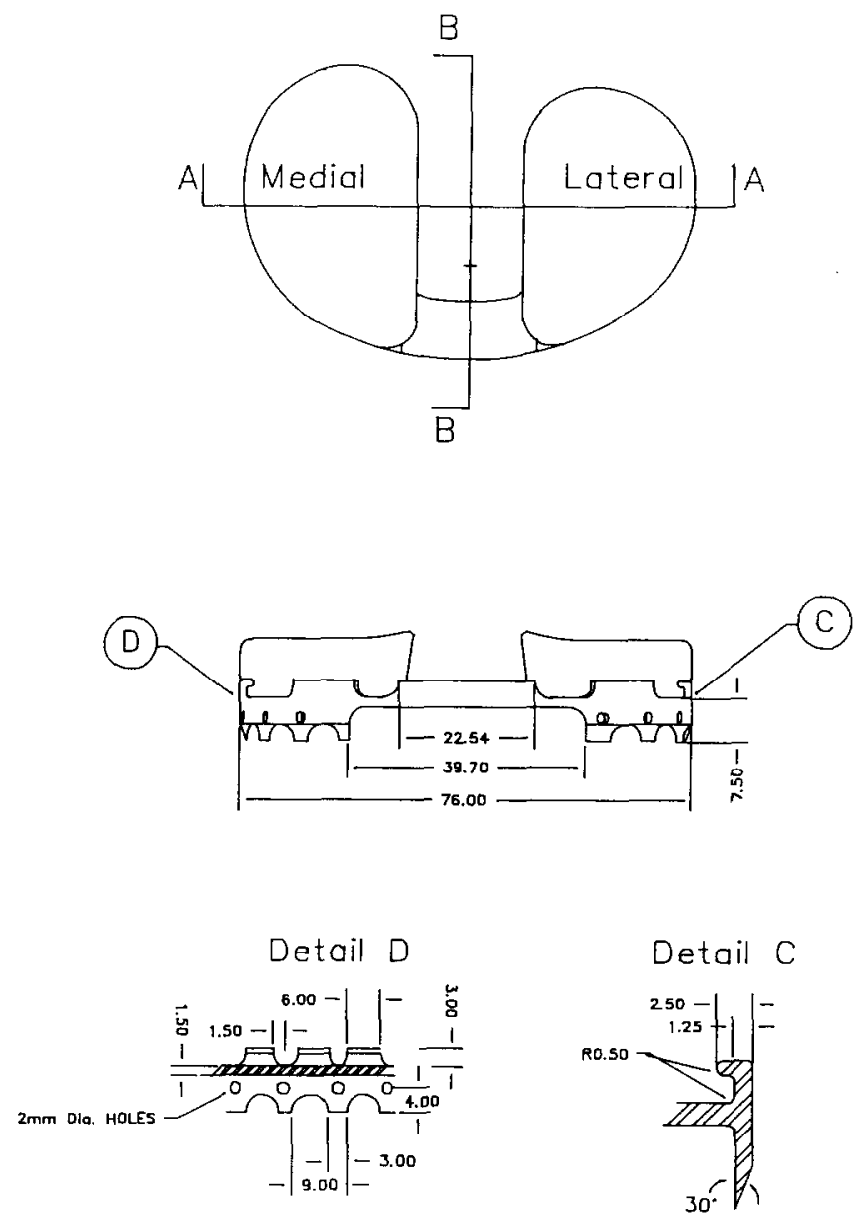

Figure 2. Dimensions of the new QUE design used in this study. Details $C$ and $D$ define the rims and knife-edge design

of the bone beyond this point was considered insignificant. The current model was made up of approximately 1400,8 -node isoparametric solid elements based upon a formulation which includes modified extra displacement shape functions aimed at improving the element behaviour when subjected to bending ${ }^{22,23}$. The element size decreased from the distal to proximal end. Individual elements were assumed to be linearly elastic, homogeneous and isotropic. Top layer node positions were modified to ensure continuity with prosthesis outlines. Because the change in subchondral bone elastic stiffness or modulus of elasticity between adjacent elements was never extremely large for areas other than the cortical bone, this was thought to be allowable. Care was taken not to alter the thickness of the cortical shell, which ranged from $1 \mathrm{~mm}$ proximally to $6 \mathrm{~mm}$ distally. Cancellous bone elastic properties were obtained from indentation tests by Little et al. ${ }^{21}$ and later confirmed by Harada et al. ${ }^{19}$. Cortical bone stiffnesses were taken from the literature $^{24}$ and ranged from $1434 \mathrm{MPa}$ for the proximal epiphyseal region to $7056 \mathrm{MPa}$ for the more distal cortex. Inhomogeneity was accounted for by employing 588 different elastic moduli measured experimentally from hardness/indentation tests ${ }^{19,21}$, varying from approximately $11 \mathrm{MPa}$ for trabecular bone to $7056 \mathrm{MPa}$ for the epiphyseal and metaphyseal shells. Poisson's ratio was assumed to be 0.3 in the entire bone model unless otherwise stated.

The geometry of the new design (QUE) was taken from the working drawings (Figures 2 and 3). The prosthesis was made up of 984 -noded quadrilateral shell elements for the base plate, in combination with 302 -noded beam elements to model the rims and knife edge. The elements within the outline of the prosthesis were generated to match the underlying bone elements. The prototype design was made of titanium alloy (Ti-6Al-4V, ASIM F136) with a Young's modulus of $117 \mathrm{GPa}$ and a Poisson's ratio of 0.3 .

The PCA geometry was taken from measurements on a large size implant (Figure 3). The model was made up of 133 4-noded quadrilateral elements for the base plate in combination with 402 -noded beam elements for the small rim surrounding the porous coating. Pegs were not modelled directly, but their influence was accounted for by generating a rigid area at the appropriate peg locations of the model. The elements within the outline were generated to match the underlying bone elements, except one node which was moved to facilitate screw fixation modelling in the proper location. This prosthesis was made from cobalt chromium alloy (ASTM F75-2) with an elastic modulus of $200 \mathrm{GPa}$ and a Poisson's ratio of 0.3 .

The interface was modelled using 105 and 142 2-noded non-linear interface elements (Stif 52 in ANSYS) for the new QUE design and the PCA model respectively. The interface elements had the capability

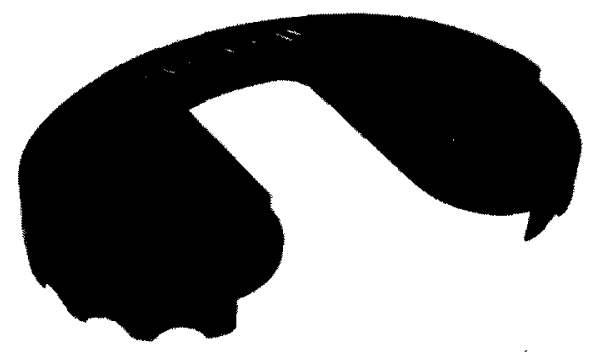

a

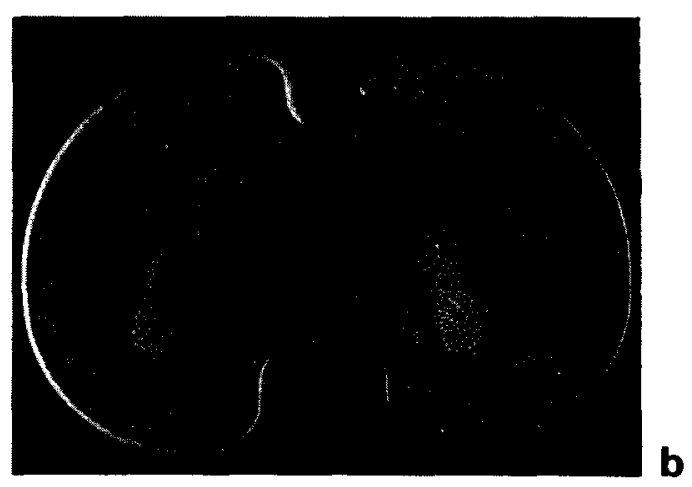

Figure 3. New QUE base plate with (a) perimeter fixation and (b) PCA tibial prosthetic base plate. 
Table 1. Current FE models studied

\begin{tabular}{lccllc}
\hline Model & $\begin{array}{c}\text { Base plate } \\
\text { thickness } \\
(\mathrm{mm})\end{array}$ & $\begin{array}{c}E \\
\text { (GPa) }\end{array}$ & $\begin{array}{c}\text { Fixation } \\
\text { type }\end{array}$ & $\begin{array}{c}\text { Screw } \\
\text { fixation }\end{array}$ & $\begin{array}{c}\text { Bone } \\
\text { Poisson's ratio }\end{array}$ \\
\hline QUE1 & 1.5 & 117 & Knife edge & No & 0.3 \\
QUE2 & 1.5 & 200 & Knife edge & No & 0.3 \\
QUE3 & 1.5 & 117 & Norims & No & 0.3 \\
QUE4 & 1.5 & 117 & Peg & No & 0.3 \\
PCA1 & 1.5 & 200 & Peg & Yes & 0.3 \\
PCA2 & 3.0 & 200 & Peg & No & 0.3 \\
PCA3 & 1.5 & 200 & Peg & No & 0.3 \\
PCA4 & 1.5 & 200 & Knife edge & Yes & 0.0 \\
PCA5 & 1.5 & 200 & Peg & \\
\hline
\end{tabular}

to model sliding with or without friction and lift-off between the prosthesis and bone surfaces. The frictionless case was considered in this study because this condition would yield the maximum relative motion at the interface similar to a clinically loosened prosthesis or initial fixation. Otherwise, an iterative solution would have been necessary for the non-linear interface elements. To limit calculation times the bone model was condensed into one superelement or substructure. This did not affect the accuracy of the results.

The bone model was fully constrained at the distal end. According to the Saint Venant's principle, this would have a negligible effect on the results at the proximal end. The influence of pegs and knife edge was accounted for by generating constraint equations which coupled the movements of nodes forming knife edge or pegs to the movement of the bone, thus enforcing the local reversible relative motion to zero.

Only an axial loading was applied, resulting in a force of $2500 \mathrm{~N}$ or approximately three times body-weight. This excluded effects of important clinical loading occurrences on, for example, the posterior part of the prosthesis, or eccentrically, more load on the medial condyle than the lateral condyle, and vice versa.

Half of the force was borne by the medial side, the other half by the lateral side. The UHMWPE load-bearing articulating surfaces were not modelled, but their effect was accounted for by determining the load redistribution to the metal base plate or backing of the tibial prosthetic component. To find the load distribution a separate model was generated of a circular UHMWPE pad, radius $12.4 \mathrm{~mm}$ and thickness $8 \mathrm{~mm}$. Young's modulus of the UHMWPE was $500 \mathrm{MPa}$, Poisson's ratio 0.3. The contact area between the femoral condyles and polyethylene pads was $120 \mathrm{~mm}^{2}$ for the medial side and $90.8 \mathrm{~mm}^{2}$ for the lateral side. The shape of the contact area was approximated by a circle. Because the exact nature of the pressure distribution on the top of the pads was unknown, several pressure distributions were applied: hemispheric (Herzian), parabolic and the distribution resulting from a uniform displacement (rigid die) on an elastic foundation. Although the resulting pressure distributions on the articular surface of the pads differed significantly, the pressure distributions at the bottom of the pads all had approximately the same shape. Only moderate differences in peak pressure and load-bearing area were indicated. Because an assumed Herzian distribution at the articulating surface was thought to be the most natural load distribution considering the shapes of common articulating prostheses, the pressure distribution resulting from it was used for the model loading on the metal backing. The peak pressure on the metal backing was approximately $9 \mathrm{MPa}$. The resulting load distribution area was approximately $344 \mathrm{~mm}^{2}$ on the medial side and $225 \mathrm{~mm}^{2}$ on the lateral side.

Apart from the method of fixation there are other parameters that would influence the reversible relative motion, such as the geometry and material properties. To investigate these influences, a series of studies were done as illustrated in Table 1 . The base plate thickness and the porous coating thickness of the PCA were each $1.5 \mathrm{~mm}$, resulting in an overall thickness of $3 \mathrm{~mm}$. In the PCA1 and PCA3-5 models it was assumed that the stiffness contribution of the porous coating was negligible, resulting in an effective thickness of $1.5 \mathrm{~mm}$. In the PCA2 model, full porous coating

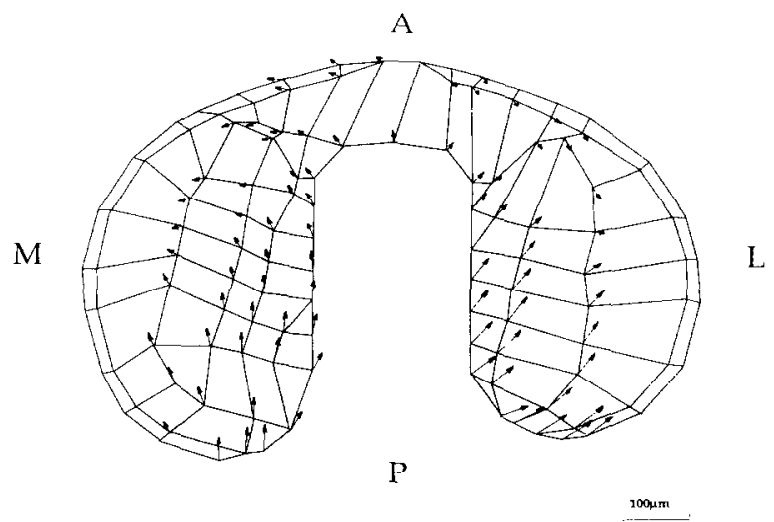

Figure 4. Vector plot of reversible relative bone motion with respect to the metal base plate in the QUE1 model. Note that in the AP direction there is a net transverse contraction of bone. 
A

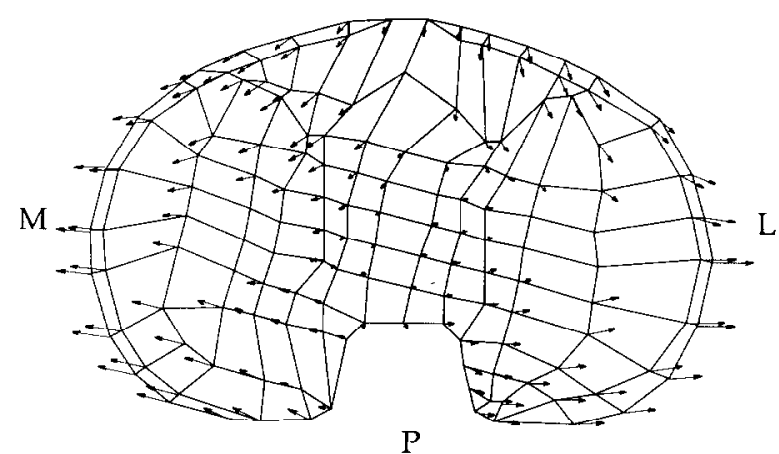

a

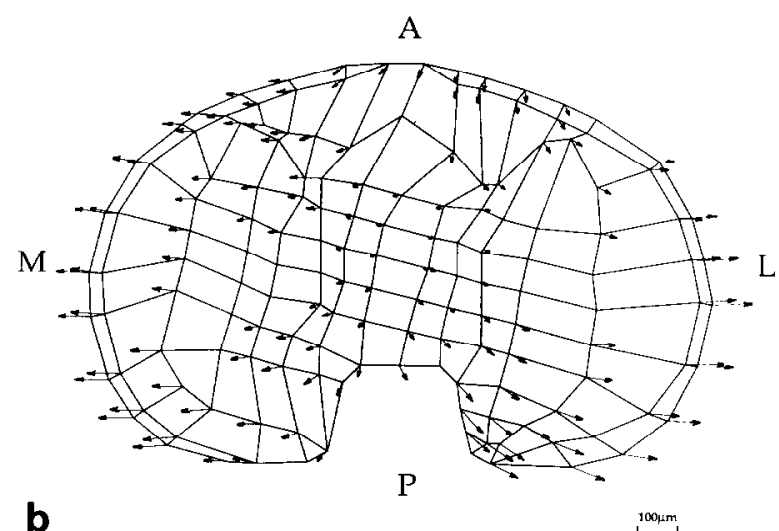

Figure 5. Reversible relative motion between the bone and metal base plate for (a) the PCA 1 model $1.5 \mathrm{~mm}$ plate thickness), and (b) the PCA2 model $(3.0 \mathrm{~mm}$ plate thickness).

stiffness contribution was assumed, resulting in an effective metal thickness of $3 \mathrm{~mm}$.

To investigate the influence of stiffness caused by the presence or absence of a cruciate retainment cut-out, models of the PCA with knife-edge fixation (PCA4) and the new design with peg fixation (QUE4) were generated.

Little is known about the Poisson's ratio of trabecular bone. Measurements on femoral trabecular bone $^{25}$ showed variation from 0.01 to 0.34 . In the original bone model, only one valuc of 0.3 was incorporated. Since the Poisson's ratio was expected to influence reversible relative motion, a bone model similar to PCA1 but with a Poisson's ratio of 0.0 was also generated as a comparison.

\section{Results}

The first calculations for the new design (QUE1) showed that at the anterior and posterior surfaces the bone was not expanding but contracting (Figure 4). This contradicted the assumption that was made when the constraint equations were generated. Originally the knife edge was connected to the bone over the full length, but the knife edge was incapable of constraining inward motions or separations with the bone. Therefore all nodes were released (i.e. gap elements were generated in those nodes) where inward motion was detected.

This transverse A-P contraction was almost non-existent in the PCA1 and PCA2 models (Figure 5a, b). The contraction was largest in the PCA5 where a Poisson's ratio of 0.0 was assigned to the bone (Figure 6) to study the sensitivity of the model.

Lift-off is not accounted for in the values of reversible relative motion mentioned below. Results showed very little lift-off in all the models under an axial compressive loading.

The absolute magnitude of the reversible relative motion was plotted along a medial-lateral mid-condylar line (Figure $7 \mathrm{a}$ ) and along posterior-anterior paths for both medial and lateral condylar areas (Figures $7 \mathrm{~b}$ and $7 \mathrm{c}$ ). The basic difference between the two modes of fixation showed up in the large relative bone motion of the PCA at the perimeter along the medial-lateral mid-condylar line (Figure 7a). In the middle of each condyle the peg fixation constrained bone motion. The flexible QUE1 design restricts the transverse relative bone motion at the perimeter and allows the bone to move elsewhere with the largest motion at the tibial eminence (edge of the central cut-out). The posterior bone in the QUE1 model showed relative motion of $25-35 \mu \mathrm{m}$ at the knife edge boundary as it was allowed to move inwards freely from the knife edge (Figures $7 \mathrm{~b}$ and $7 \mathrm{c}$ ). The PCA models showed large reversible relative motion at the perimeter, a sharp decrease to zero as the path intersected through the peg areas and limited reversible relative motion between the pegs. The difference between the PCA1 and PCA2 models was relatively small.

The QUE1 model showed much smaller overall relative reversible motion except in the central areas where, in the PCA1 and PCA2 models, the pegs had a strong restraining effect. Along the posterior-anterior paths, the value of bone motion for all models was smaller at the antcrior surface than at the posterior surface.

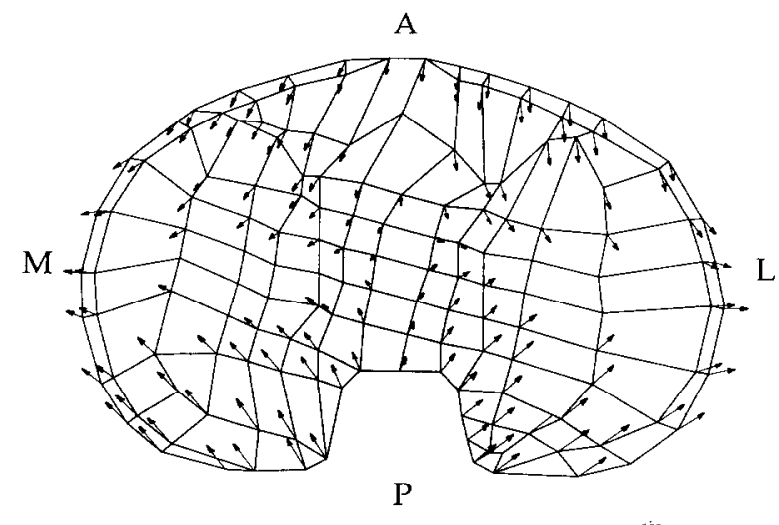

Figure 6. When the bone was assigned a Poisson's ratio of 0.0 rather than 0.3 , reversible relative bone motion increased significantly in the A-P direction (compared with Figure 5). 

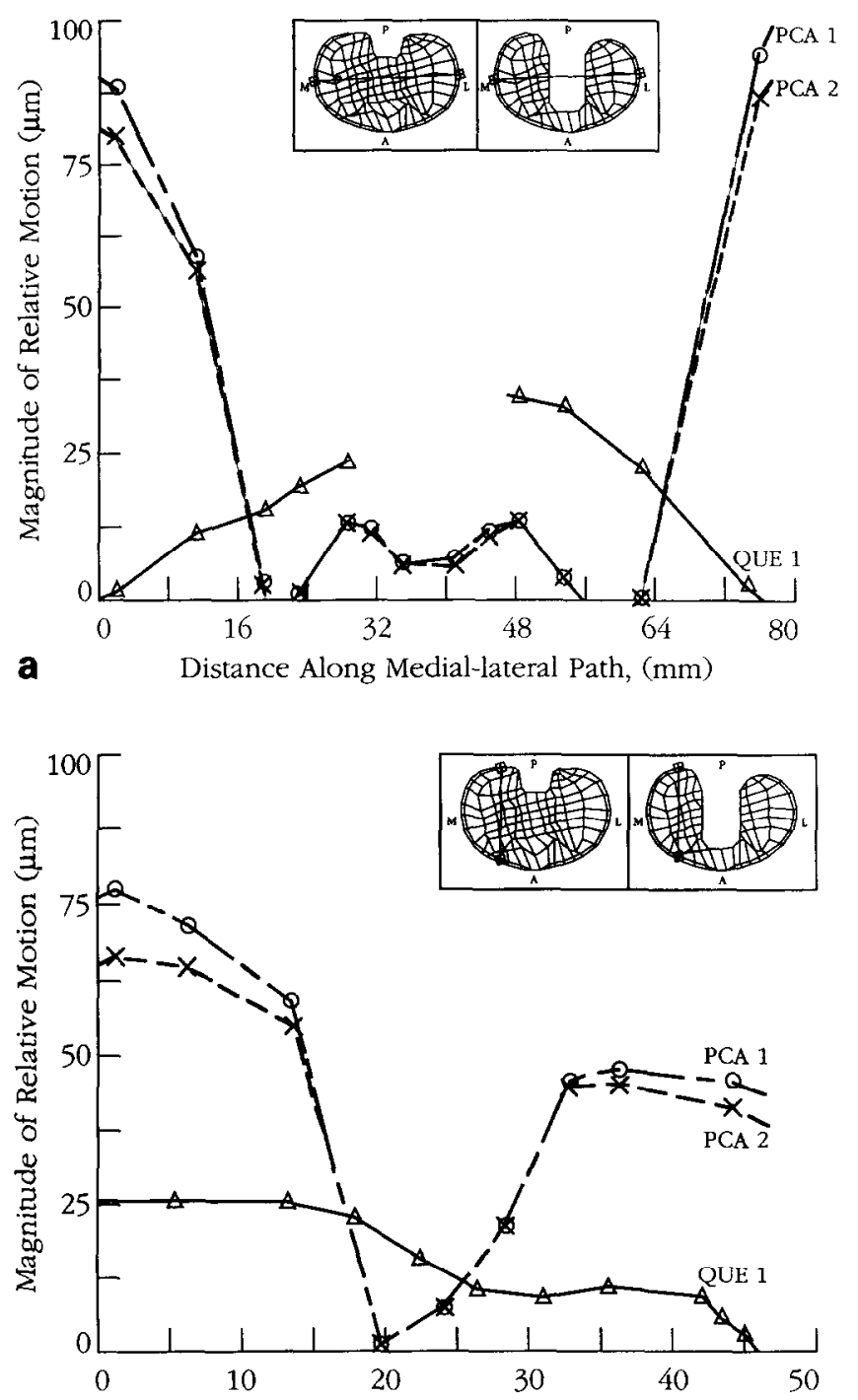

b Distance Along Posterior-anterior Path, medial side (mm)

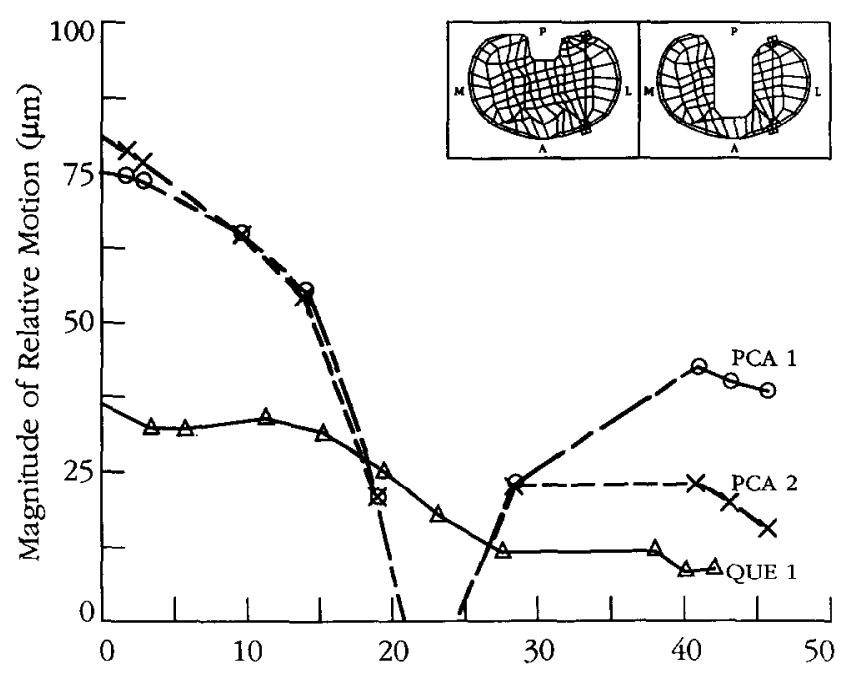

C Distance Along Posterior-anterior Path, lateral side (mm)

Figure 7. Plots of relative bone motion as a function of location along (a) a mid-tibial mediolateral path; (b) a medial mid-condylar path, and (c) a lateral mid-condylar path. See insets for reference. The displacement at each element intersection was calculated as a linear interpolation from the relevant nodal points.
Figure 8 shows the axial displacement of the prostheses due to loading. All cases indicated tilting with the posterior part sunk more than the anterior and the lateral side lower than the medial side. The plots also indicate metal plate flexing under load.

Average and maximum relative reversible motion was calculated for all models. Average motion was defined as the sum of all relative displacements (absolute magnitudes) as calculated for the gap elements, divided by the total number of gap elements. Zero motion as a result of constrained nodes was not counted. The results are summarized in Table 2 .

Stiffer models with the same fixation method predicted less reversible relative motion. This is in accordance with findings in the literature ${ }^{16.26}$. The values for QUE1, QUE2, QUE3, and PCA4 showed the effect of knife edge perimeter fixation and the difference from the models not having a perimeter knife edge was mechanically significant; however vigorous statistical analyses was not pursued.

\section{Discussion}

The numerical data presented in this study should bè used for qualitative comparisons of one prosthesis design concept over the other. Absolute values were affected by element mesh density, simplifications in geometry, bone material characterization and joint loading.

Other model characteristics which give some indication of model accuracy and validity are model and total element strain energy, sums of the applied and reactive forces, sums of element forces (product of normal stress and element surface area) at selected levels, and the continuity of nodal displacements across element boundaries. All of these aspects behave well in the present model.

Element density was insufficient to give accurate stresses in any specific area of the implant and bone. Specific local displaeements were similarly affected but global displacement patterns gave a clear insight into the differences in performance between the new QUE design and a commercial tibial implant (PCA).

The bone model geometry was developed from an average-sized tibia judged to be normal (free from malformation) by orthopaedic surgeons participating in our research. There are obvious differences in tibial geometry and bone material properties between persons. Here, the same bone model was used in all comparisons, thus excluding individual bone variations from the study.

Heterogeneous distribution of cancellous bone stiffness was considered the most important factor in load-bearing analysis ${ }^{21}$. Therefore, in principle each element in our model representing cancellous bone was assigned its own stiffness value. Cortical bone values represented the distinct elasticity values of the epiphyseal, metaphyseal, and diaphyseal regions. The cortical shell in the epiphyseal area (the $10-20 \mathrm{~mm}$ most proximal part) was essentially kept at the same 

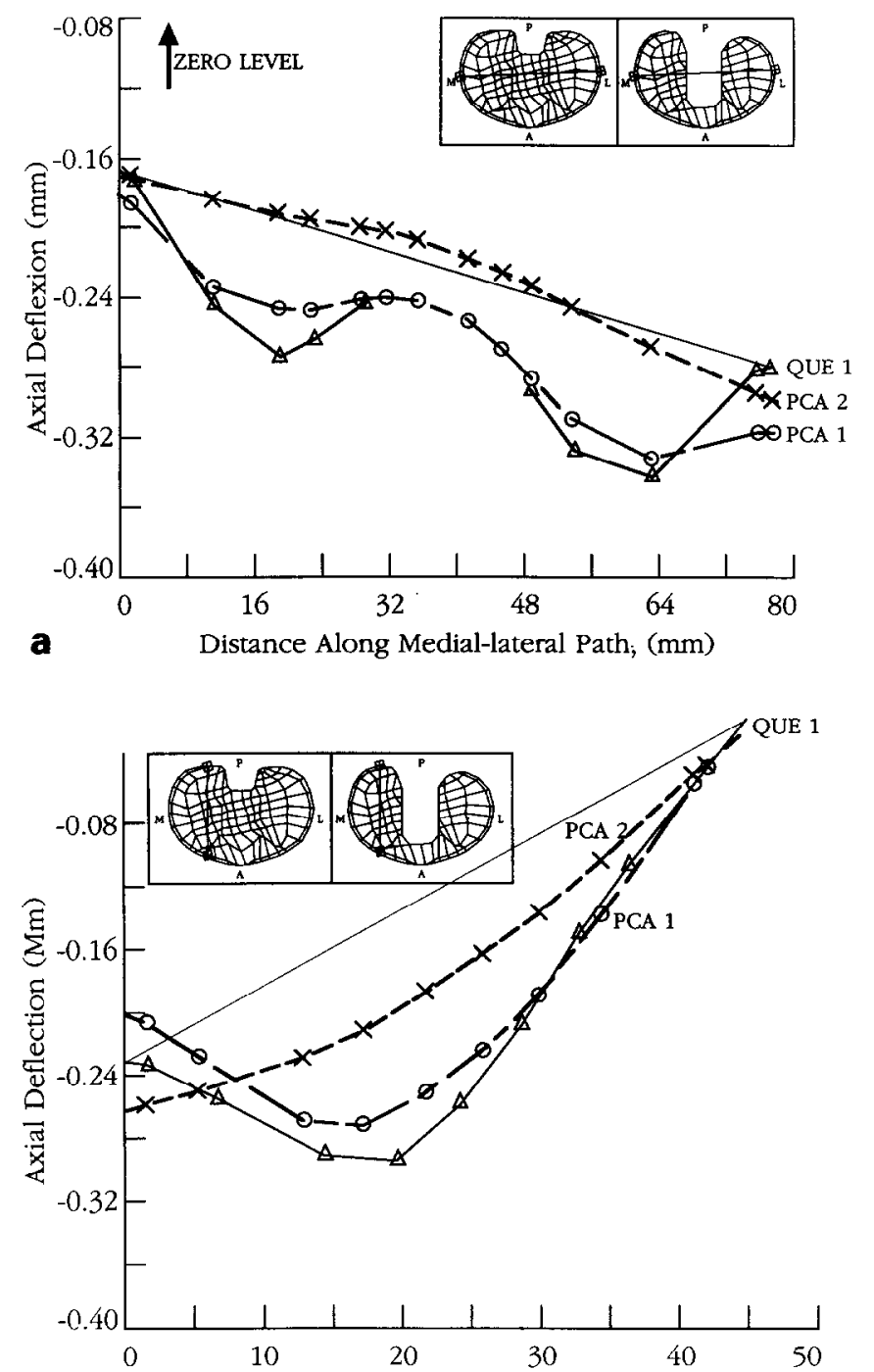

b Distance Along Posterior-anterior Path, medial side ( $\mathrm{mm}$ )

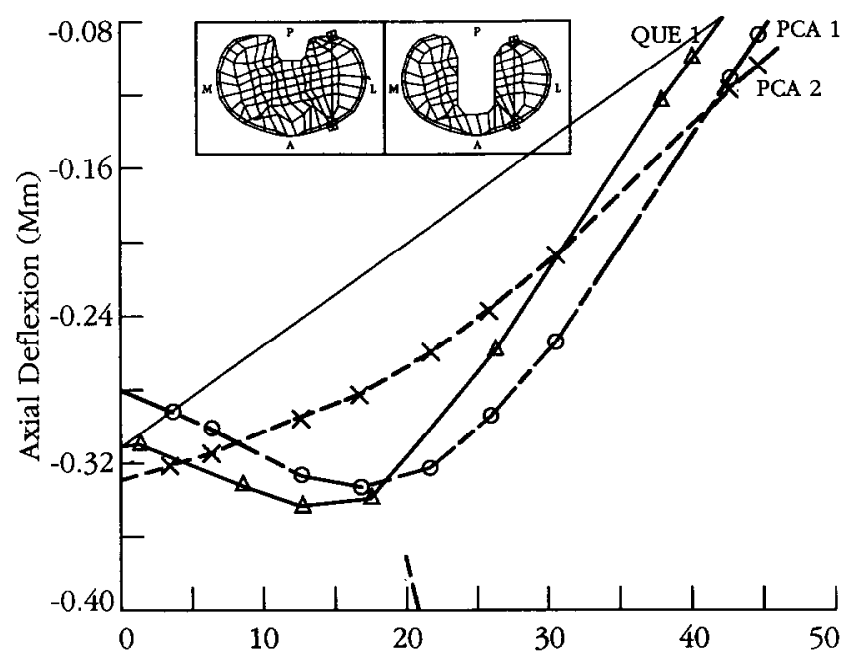

C Distance Along Posterior-anterior Path, lateral side ( $\mathrm{mm}$ )

Figure 8. Axial deflexion along (a) mediolateral and (b, c) postero-anterior paths as identified by the insets. Tilting of the mediolateral and postero-anterior axes is clearly shown. Flexion or 'cupping' under load is most visible for the thinner prostheses. Note that deflexion is measured downwards (distally) from the zero level (not shown in the graphs). The continuous thin straight line represents rigid body motion of the new design (zero deflexion).
Table 2. Average and maximum magnitudes of reversible relative motion. Bold models QUE1 and PCA1 represent actual designs

\begin{tabular}{llc}
\hline Model & Average $(\mu \mathrm{m})$ & Maximum $(\mu \mathrm{m})$ \\
\hline QUE1 & 17 & 37 \\
QUE2 & 15 & 42 \\
QUE3 & 19 & 45 \\
QUE4 & 37 & 90 \\
PCA1 & 44 & 101 \\
PCA2 & 41 & 90 \\
PCA3 & 47 & 100 \\
PCA4 & 13 & 34 \\
PCA5 & 44 & 77 \\
\hline
\end{tabular}

Note: For variation in the reversible relative motion at each nodal point, see Figures 4-6. Also, the average motion is a deterministic value and is not meant as a statistically relevant value.

value as the hardest and stiffest cancellous bone. However, anisotropy was not accounted for as its effect is not an overriding one ${ }^{27}$. Also, for the cortical bone, the anisotropy had little effect if loading was directed along the long axis of the bone, as was the case in this study $^{28}$. The bone was considered linearly elastic with values for the cancellous bone derived from hardness testing ${ }^{19,21}$ and values for cortical bone from the literature ${ }^{24}$. It was assumed that linear elasticity was valid at physiological loading rates ${ }^{29}$.

\section{Knife edge versus peg fixation}

From the results of the axial loading case, the knife edge provided better resistance to reversible relative motion than peg fixation in all the models examined. The difference in average reversible relative motion between a prosthesis with peg fixation and one with a knife-edge fixation was minimally $49 \%$. The difference peaked at $77 \%$ between Models PCA3 and PCA4. Both models had an assumed thickness of $1.5 \mathrm{~mm}$ with PCA peg fixation in the PCA3 and perimeter knife edge fixation in PCA4.

The largest amount of bone movement in the peg fixation models took place at the medial and lateral perimeters. This was also the area where the knife-edge design eliminated reversible relative motion, explaining the effectiveness of the knife-edge fixation. In effect, the knife edge forced bone and prosthesis to expand together under load.

If relative bone motion impedes bone ingrowth, it should be suppressed, particularly at the perimeter where maximal fixation strength is needed to counteract rotation, tilt, and shear of the prosthesis. The new design appeared to be highly effective in suppressing transverse bone motion under axial loading.

The reversible relative motion between the pegs of models PCA1 and PCA2, and at the perimeter, was comparable with in-vitro studies by Tissakht et al. ${ }^{13}$.

Prosthesis flexibility, especially out of plane, is 
thought to be important for physiological load transfer, as the prosthesis design attempts to mimic the subchondral bone plate it replaces. Increased stiffness of the prosthesis decreased relative reversible motion but only slightly, as shown in the comparison between the PCA1 $(1.5 \mathrm{~mm})$ and PCA2 $(3 \mathrm{~mm})$ models (Figure 7).

The influence of in-plane stiffness became clear when the QUE2 and the PCA4 were compared. Both models had the same fixation method (with knife edge), the same Young's modulus of $200 \mathrm{Gpa}$, and the same base plate thickness of $1.5 \mathrm{~mm}$, but a very different in-plane stiffness, because of the presence or absence of a cruciate retainment cut-out. The decrease in stiffness caused by the cut-out resulted in a $15 \%$ higher average reversible relative motion. This might be an indication that when possible (in the absence of cruciate ligaments) a prosthesis with a higher in-plane stiffness, and therefore without a deep cut-out, should be used.

Figure 8 shows a cupping effect under load of the 1.5 mm-thick prosthesis models (QUE1 and PCA1). When the porous coating was assumed to be fully contributing to the metal stiffness of the prosthesis (PCA2), the cupping effect almost disappeared. It is unclear how a composite metal plate of $1.5-\mathrm{mm}$ cast $\mathrm{Co}-\mathrm{Cr}$ alloy with $1.5-\mathrm{mm}$ sintered beads would react under load with respect to flexural stiffness. If the beads do stiffen the prosthesis, they are therefore subjected to high inter-bead stresses. If on the other hand the effect of the beads were negligible, the beads would have zero transverse interaction in flexion. The latter is only conceivable if the bead network had been broken in fatigue, or transverse bonding was never fully developed. It is therefore realistic to assume that the PCA prosthesis had an actual stiffness between these extremes as described in models PCA1 and PCA2.

Deflexions of 160 to $340 \mu \mathrm{m}$ in the proximal-distal direction (Figure 8 ) include deformation of the $100-\mathrm{mm}$ tibial bone under load. A line connecting the medial and lateral margins of the QUE1 model and the PCA1 and PCA2 models would indicate relative displacement values of the metal tray itself. The same method of referencing would be of use in the posterior-anterior direction. This indicated a cupping effect under load medially of approximately $120 \mu \mathrm{m}$ for the new design (QUE1) as measured from the posterior-anterior reference line (Figure $8 \mathrm{~b}$ ). This cupping is important as it promotes load sharing by the cancellous bone with the epiphyseal and metaphyseal shell.

The influence of an anterior screw used in PCA fixation on reversible relative motion was small; the decrease in average reversible relative motion was $7 \%$. This was explained by the fact that the screw is placed in what was almost a plane of symmetry of relative reversible motion as was illustrated in Figures 4-6. However, the screw has a function in out-of-plane initial fixation that was not included in this study.

The influence of the Poisson's ratio on the average reversible relative motion was small. Although Figure 6 showed a clear change in localized reversible relative motion magnitude and direction, the average magnitude stayed virtually the same when the Poisson's ratio was changed from 0.3 (PCA1) (Figure 5a) to 0.0 (PCA5) (Figure 6). The maximum value of reversible relative motion, however, decreased by $24 \%$ (Table 2 ).

The posterior to anterior contraction of the bone was probably caused by the specific tibial geometry, i.e. the widening or transverse flaring of the tibia proximally, which will contribute the mediolateral widening as a result of bending under load with anterior-posterior contraction occurring at the same time. The overall expansion of bone as a result of Poisson's effect would compensate for the anterior-posterior contraction. When the Poisson's ratio was changed to zero, the expansion due to the Poisson's effect was thereby eliminated and only the effect of the specific geometries on bending expansion as a result of tibial mediolateral bending was left. This led to significant posterior-anterior contraction in the PCA5 model, which was, apart from the Poisson's ratio of the bone, identical to the PCA1 model.

Apart from possible influence of local material properties, tilting was also caused by the specific bone geometry with the proximal flaring out and widening of the tibial bone on the posterior side. The posterior-anterior tilt was between 180 and $230 \mu \mathrm{m}$ as measured from the highest anterior point in Figures $8 \mathrm{~b}$ and $8 \mathrm{c}$. The medial-to-lateral tilt was approximately $120 \mu \mathrm{m}$ as measured from Figure $8 \mathrm{a}$.

It should be noted that the phenomena of the contraction of bone and the tilting of the prosthesis could not have been revealed in a $2 \mathrm{D}$ or axisymmetric analysis, thus supporting the choice of $3 \mathrm{D}$ models in our study.

In conclusion, it has been shown that reversible relative bone-prosthesis motion is sensitive to the mcans of mechanical fixation of the prosthesis in knee arthroplasty. Only lately are quantitative data published on micromotion threshold values for bone ingrowth. Burke et al. ${ }^{30}$ reported a threshold of $40 \mu \mathrm{m}$, below which bone ingrowth took place. Pilliar et al. ${ }^{11}$ has evidence that $25 \mu \mathrm{m}$ is the threshold value. Both values indicate that a perimeter fixation as shown in the QUE1 model appears to maintain micromotion values below the threshold.

\section{Acknowledgements}

This research was made possible by a grant from the Natural Sciences and Engineering Research Council (NSERC) of Canada. Contributions by members of the Clinical Mechanics Group at Queen's University, the Kingston General Hospital, and the University of Twente are gratefully acknowledged.

\section{References}

1 Cheal EJ, Hayes WC, Lee CH et al. Stress analysis of a condylar knee tibial component: influence of metaphyseal shell properties and cement injection depth. $J$ Orthop Res $1985 ; 3: 424-34$ 
2 Ahmed AM. Characterization and improvement of the metal/cement interface: an overview. In: Lewis JL, Galante IO. eds. The Bone-Implant Interface, Workshop Report. Chicago: American Academy of Orthop Surgeons 1983, 102-10

3 Hungerford DS, Kenna RV, Krackow KA. The porous coated anatomical knee. Orthop Clin North Am 1982: 13(1): 103-22

4 Teske CA, Mayor MB, Collier JP, Suprenant VA. A comparative study of the effectiveness of plasma sprayed hydroxyapatite and tricalcium phosphate coatings in enhancing the fixation of smooth Ti-6Al-4V implants. 35th Annual Meeting of the Orthopaedic Research Society, Las Vegas, Nevada 1989: 333

5 Cook SD, Barrack RL, Thomas KA, Haddad RJ. Quantitative histologic analysis of tissue growth into porous total knee components. J Arthroplasty, 1989; [Suppl]: 33-43

6 Ryd L. Micromotion in knee arthroplasty; a rocntgen stereophotogrammetric analysis of tibial component fixation. Acta Orthop Scand 1986; [Suppl 220]: 11-40

7 Carter DR. Mechanical loading history and skeletal biology. J Biomech 1987; 20(11/12): 1095-109

8 Lanyon LE. Functional strain in bone tissue as an objective, and controlling stimulus for adaptive bone remodelling. J Biomech 1987; 20(11/12): 1083-93

9 Lewis JL, Askew MJ, Jayeox DP. A comparative evaluation of tibial component designs of total knee prostheses. J Bone Joint Surg 1982; 64A (1): 129-35

10 Ducheyne P, DeMeester P, Aernoudt E et al. Influence of a functional dynamic loading on bone ingrowth into surface pores of orthopaedic implants. J Biomed Mater Res $1977 ; 11(6): 811-38$

11 Pilliar RM, Lee JM, Maniatopolous C. Observations on the effect of movement on bone ingrowth into poroussurfaced implants. Clin Orthop Rel Res 1986; 208: 109-13

12 Ryd L, Lindstrand A, Stenstrm A, Selvik G. Porous coated anatomic tricompartmental tibial components; the relationship between prosthetic location and micromotion. Clin Orthop Rel Res 1990; 251: 189-97

13 Tissakht M. Ahmed AM, Mulas G. Measurement of bone/prosthesis interface relative motion: comparison with analytical predictions. 10th Annual Conference of the Canadian Biomechanics Society, McGill University, Montreal, Quebec. 1989: 39-42

14 Huiskes R, Chao EYS. A survey of finite element analysis in orthopaedic biomechanics: the first decade. $J$ Biomech 1983: 16(6): 385-409

15 Yettram AL. Effect of interface conditions on the behavior of a Freeman hip endoprosthesis. J Biomech Eng 1989; 11: 520-24

16 Shirazi-Adl A, Ahmed AM. A parametric model study on the interface movements in porous surfaced tibial implants. Ann Biomed Eng 1989; 17(4): 411-21

17 Strickland AB, Natarajan R, Andriacchi TP. Transverse motion at the interface between the tibial component and bone in a total knee replacement: an analytical model. AMD (Symposia Series) (American Society of Mechanical Engineers, Applied Mechanics Division), Biomechanics Symposium. Third Joint ASCE/ASME Mechanics Conference, New York 1989; 98: 285-8

18 Natarajan R, Andriacchi TP. New analytical method for the analysis of bone ingrowth in porous tibial components. ASME Biomechanics Symposium, San Diego 1989;281-4

19 Harada Y, Wevers HW, Cooke TDV. Distribution of bone strength in the proximal tibia. J Arthroplasty 1988; $3(2): 167-75$

20 Goldstein SA. The mechanical properties of trabecular bone: dependence on anatomic location and function. J Biomech 1987; 20: 1055-61

21 Little RB, Wevers HW, Siu D, Cooke TDV. A three dimensional finite element analysis of the upper tibia. J Biomed Eng 1986; 108: 111-9

22 Wilson EL, Taylor RL, Doherty WP, Ghaboussi J. Incompatible displacement models. In: Fenves SJ et al. eds. Numerical and Computer Methods in Structural Mechanics. Academic Press Inc., New York, London 1973; 43-57

23 Taylor RL, Beresford PJ, Wilson EL. A non-conforming element for stress analysis, Int J Numer Methods Eng 1976; 10: 1211-19

24 Murray RP, Hayes WC, Edwards WT, Harry JD. Mechanical properties of the subchondral late and the metaphyseal shell. 30th Annual Meeting of the Orthopaedic Research Society, Atlanta, Georgia, 1984; 197

25 Knauss P. Material properties and strength behaviour of spongy bone tissue at the coxal human femur. Biomed Tech Berlin 1981; 26(9): 200-10

26 Rohlmann A, Cheal EJ, Hayes WC, Bergmann G. A nonlinear finite element analysis of interface conditions in porous coated hip endoprosthesis. J Biomech $88 ; 21(7)$ : 605-11

27 Askew M, Lewis J. Analysis of model variables and fixation post length effects on stresses around a prosthesis in the proximal tibia. J Biomech Eng 1981; 103: 239-45

28 Huiskes R, Janssen JD, Slooff TJ. Detailed comparison of experimental and theoretical stress-analyses of a human femur. ASME-ASCEW Applied Mechanics Fluids Engineering and Bioengineering Conference, New York, 1981; 45: 211-34

29 Pugh JW, Rose RM, Radin EL. A structural model for the mechanical behavior of trabecular bone. $J$ Biomech $1973 ; 6: 657-70$

30 Burke, DW, Bragdon CR, O'Connor DO et al. Dynamic measurements of interface mechanics in vivo and the effect of micromotion on bone ingrowth into a porous surface device under controlled loads in vivo. Transactions of the 37th Orthopaedic Research Society Meeting 1991: 16, Section 1: 103 